\title{
Sistemas de documentación en línea para aplicaciones Java
}

\author{
Ricardo Eíto Brun \\ Universidad de Granada \\ Facultad de Biblioteconomía y Documentación
}

\subsection{Resumen}

El reconocimiento que ha logrado el lenguaje Java y su adopción por parte de los desarrolladores de software ha conllevado la necesidad de dotar a los programas escritos en este lenguaje de sistemas de documentación y ayuda en línea que ofrezcan características similares a los sistemas utilizados en el entorno Windows. Se analizan las alternativas disponibles actualmente para crear y gestionar documentación en línea para aplicaciones escritas en lenguaje Java. Como conclusión se señala la necesidad de que los sistemas actuales alcancen la madurez de los sistemas disponibles para Windows.

Palabras clave: Java. Documentación en línea.

\subsection{Abstract}

The acknowledgement that Java has got and its adoption by many software developers has brought a need to provide Java programs with online documentation and help similar to those used in the Windows environment. The different alternatives existing in the market for creating and managing online documentation for Java programms are analyzed. It is concluded that existing systems are yet to reach the maturity of Windows based systems.

Keywords: Java. Online documentation.

\section{Introducción}

Uno de los campos menos explotados por los profesionales de la documentación es el diseño, creación y gestión de los sistemas de documentación en línea, o de las ayudas, que acompañan a los programas informáticos.

La documentación en línea consiste en instrucciones escritas, distribuidas en formato electrónico, que permiten obtener las máximas prestaciones de las aplicaciones software. 
Si bien puede parecernos que estos sistemas son algo ajenos a la orientación profesional a la que estamos acostumbrados, deberíamos desechar esta idea y analizar con más detenimiento las posibilidades que abre este sector.

La documentación de software permite:

- Minimizar los costes de los centros de soporte técnico, que a su vez repercuten en los usuarios y fabricantes de aplicaciones .

- Permitir la autoformación de usuarios.

- Minimizar los errores en la interacción del usuario con las aplicaciones informáticas.

Por otra parte, si nos centramos en aplicaciones destinadas a la gestión de procesos empresariales o administrativos, la documentación en línea llega a desempeñar un papel aún más importante, ya que las directrices que establece documentan y permiten homogeneizar los procedimientos de trabajo soportados por las aplicaciones informáticas.

Los puntos anteriores son aplicables a cualquier tipo de documentación técnica (impresa o no). La documentación en línea une a las ventajas anteriores las que nos ofrece el documento en formato digital:

- Mejores mecanismos de acceso a la información mediante búsquedas en texto completo, por palabras clave, tablas de contenidos jerárquicas desplegables, etc.

- Mínimos costes de distribución.

- Facilidad para personalizar y parametrizar la documentación mediante anotaciones, marcas de lectura — bookmark- e incluso la propia edición y modificación del texto, y ...

- Mayor rapidez y flexibilidad en la distribución de actualizaciones del producto, patches, mejoras y errores corregidos, etc.

- Reducción del tiempo requerido para publicar.

La principal característica de un sistema de documentación en línea para aplicaciones software - $y$ desde nuestra perspectiva, uno de los factores críticos para poder designar con este nombre a un sistema- es la vinculación del contenido informativo del hiperdocumento con la presentación de la aplicación, de forma que, en cualquier momento, el usuario pueda obtener la información que explica la parte de la aplicación con la que está trabajando.

Se utiliza el término 'sensibilidad al contexto' para denominar a esta característica. En este trabajo no se considerarán los sistemas de edición electrónica que no cumplen esta característica, y que podríamos definir como libros electrónicos o manuales en formato digital. 


\section{Evolución de los sistemas de documentación en línea.}

Los primeros sistemas de ayuda en línea se escribieron para aplicaciones que se ejecutaban en sistemas operativos en modo texto (MS-DOS, Unix, mainframes, etc.). Estos sistemas solían estar basados en lenguajes de marcas próximos al SGML — e incluso al más reciente XML—y aportaban capacidades hipertextuales, sensibilidad al contexto, etc.

Con la evolución de la informática de sobremesa a entornos gráficos más amigables - y especialmente con la llegada de los ordenadores Macintosh y Microsoft Windows-, surge la posibilidad de diseñar sistemas de documentación en línea más sofisticados. De hecho, pronto estuvieron disponibles entornos para su creación y gestión.

El hito más importante en esta evolución es la publicación de la versión 3.0 del sistema operativo Microsoft Windows, que integraba el sistema de ayudas WinHelp.

Entre las características más importantes de WinHelp se encontraba su libre distribución - royalty free —, que unida a la popularidad alcanzada por este sistema operativo le convirtió en poco tiempo en un punto de referencia obligado para otros sistemas de edición electrónica.

De hecho, WinHelp pronto superó las expectativas que se cifraron en él inicialmente, para pasar a convertirse en una alternativa válida en proyectos de edición electrónica más ambiciosos, cuya finalidad no se cifraba únicamente en proveer instrucciones escritas para la utilización de una aplicación software.

Entre las características de Winhelp destacaba:

- Generación de hipertextos a partir de un documento en formato RTF.

- Posibilidad de definir índices de palabras clave que representasen el contenido de los distintos temas o páginas del hiperdocumento.

- Sensibilidad al contexto desde el cual se solicitaba la información.

- Posibilidad de personalizar el visor del hiperdocumento mediante macros simples.

- Capacidad de mostrar contenidos multimedia.

- Incorporación de mecanismos de edición básicos (anotaciones y bookmarks).

- Soporte a gráficos sensitivos, es decir, gráficos con zonas delimitadas que actuaban como origen de un hiperenlace.

Para crear hiperdocumentos era necesario trabajar con documentos escritos en formato RTF (desarrollado por Microsoft) e imágenes en formato BMP o WMF. Los títulos de cada página, las palabras clave y los números para las lla- 


\section{4}

madas contextuales se tenían que codificar mediante notas a pie de página en el documento RTF.

Tras preparar el texto era necesario crear un archivo de proyecto (consistente en un archivo en texto plano con extensión .hpj), y compilar estos dos archivos con una herramienta distribuida gratuitamente por Microsoft. Como resultado se obtenía un único archivo con extensión .hlp que podría distribuírse. Todas las versiones de Microsoft Windows incorporaban un visor capaz de mostrar estos archivos.

Dada la dificultad de crear y mantener un documento en formato RTF con todas sus notas a pie de página, distintos fabricantes de herramientas software comenzaron a ofrecer comercialmente entornos gráficos que facilitaban este trabajo. Entre ellos destaca Blue Sky Software, fabricante del popular RoboHelp, ForeFront, HDK, Doc-To-Help o Software Interphase.

\section{Compatibilidad de WinHelp}

Si bien WinHelp se diseñó para funcionar exclusivamente en aplicaciones que se ejecutasen en el sistema operativo Windows, otros fabricantes desarrollaron sistemas que garantizaban la compatibilidad y la posibilidad de usar estos hiperdocumentos en otras plataformas. Por ejemplo, HyperHelp diseñó un sistema que generaba sistemas de ayuda para máquinas Unix y Digital OpenVMS a partir de archivos RTF, HPJ y BMP, aunque también reconocía textos escritos en formato MIF (propietario de FrameMaker) y SGML.

Quick Help-Altura desarrolló un módulo que convertía hiperdocumentos HLP al formato de ayuda propio de los ordenadores Macintosh, logrando unos niveles de compatibilidad próximos al $90 \%$.

\section{Windows 95 y WinHelp 4.0}

El segundo hito importante en el desarrollo de estos sistemas es el lanzamiento del sistema operativo Microsoft Windows 95. Este programa se acompañó de una nueva versión de WinHelp, la 4.0, que aportaba mejoras sustanciales frente a la versión anterior, entre otras:

- Tabla de contenidos desplegable para facilitar la navegación por la estructura del hiperdocumento y contextualizar la posición del hiperdocumento en la que se encontraba el usuario en cada momento.

- Búsqueda en texto completo, gracias a un índice con extensión .fts creado automáticamente en el momento de compilar el archivo.

- Mayor interactividad con la aplicación para la que se diseñaba el hiperdocumento gracias a la macro Shortcut. Esta macro permite enviar mensajes

Scire. $4: 1$ (en.-jun. 1998) 131-145. 
a Windows desde botones embebidos en el documento. De esta forma se pueden desencadenar acciones que implementaban el interface del programa: selección de opciones de menú, clicks sobre botones, etc.

- Enlaces dinámicos, mediante las macros k-link y a-link.

El sistema de producción que se tenía que utilizar con WinHelp 4.0 no se diferenciaba del de las versiones anteriores. Únicamente se tenía que trabajar con una versión diferente del compilador, que Microsoft continuó distribuyendo gratuitamente. El visor de hiperdocumentos se incluía sin coste alguno en el sistema operativo Windows 95.

\section{Sistemas basados en HTML}

Finalmente, el último hito que debemos destacar es la aparición de los sistemas basados en HTML. Su característica principal es que toman como base textos escritos en HTML en lugar de textos en formato RTF.

Estos sistemas se anunciaron oficialmente el 7 de febrero de 1996 en la WinHelp Conference celebrada en Seattle. La asociación profesional WinWriters organiza anualmente estos encuentros profesionales.

Se presentaron dos sistemas: Microsoft HTML-Help y Netscape NetHelp, que ofrecían las siguientes ventajas:

- Carácter abierto y multiplataforma: un mismo hiperdocumento podría ser leído por cualquier usuario equipado con un navegador capaz de interpretar HTML estándar, con independencia del tipo de ordenador que estuviese utilizando (PC Wintel, terminal X-Motif o Macintosh).

- Mayores posibilidades de edición y personalización.

- Acceso universal a la documentación en línea y ...

- Posibilidad de distribuir el hiperdocumento entre distintas sedes locales y remotas, a las que se podría acceder mediante el protocolo HTTP (1).

Sin embargo, aunque las propuestas de Microsoft y Netscape compartían una base común — el lenguaje HTML — , presentaban una serie de problemas:

- Incompatibilidad entre los dos sistemas, e incluso entre estos sistemas y el lenguaje HTML estándar. Si NetHelp hacía uso intensivo de la tecnología JavaScript, Microsoft HTML-Help se basaba en la utilización de controles ActiveX, en la especificación SiteMap (propuesta por Microsoft al World Wide Web Consortium como un posible estándar) y en un formato compilado propietario.

- Esta incompatibilidad obligaba a disponer de un navegador de Internet específico para poder acceder a los hiperdocumentos creados con cada tipo 
de sistema: Netscape Navigator para NetHelp y Microsoft Internet Explorer para HTML-Help.

- Además, cada sistema implementaba de manera distinta la sensibilidad al contexto, es decir, la forma en la que los progra madores debían llamar al sistema de ayuda desde sus aplicaciones.

A estos problemas se tienen que añadir las limitaciones propias del lenguaje HTML:

- Imposibilidad de buscar en el texto completo de los documentos,

- Restricciones en las posibilidades de formateo (en aquel entonces no se había hecho pública la especificación Cascading Style Sheets) y ...

- Un desarrollo lento e interrumpido, impropio de los ritmos de desarrollo característicos de los programas de edición electrónica comerciales.

A pesar de estos problemas, en Seattle se confirmó un hecho inevitable y a todas luces evidente: el lenguaje HTML y los protocolos abiertos de Internet se habían convertido en un elemento de valor estratégico para el desarrollo de aplicaciones y, por lo tanto, para el diseño de su documentación.

\section{El lenguaje Java}

Java ha supuesto un cambio de paradigma en el desarrollo de aplicaciones informáticas. No se trata tan sólo de un lenguaje de programación. Más aún, se ha convertido en una plataforma para el desarrollo de código portable e independiente de plataformas hardware específicas. Al mismo tiempo, su presencia se está haciendo más y más común al margen de las aplicaciones software, y empezamos a oir hablar de tarjetas inteligentes e incluso electrodomésticos basados en este lenguaje.

Java es más fácil que el popular $\mathrm{C}++$, ofrece capacidad multiproceso y las ventajas propias de la orientación a objetos: reutilización de código, mínimo tiempo de desarrollo, seguridad, etc.

Además, está optimizado para ejecutarse en Internet, con lo que hace realidad la promesa del acceso universal al software y la distribución de aplicaciones a través de la Red.

Todos estas características potenciaron la gran aceptación y entusiasmo con el que se recibió a este lenguaje. Actualmente tenemos la certeza de que el número de aplicaciones escritas en Java aumentará, y con ello, la necesidad de proporcionar documentación en línea. Pero... ¿de qué alternativas disponemos?

La situación actual la podemos definir como una situación de vacío, ya que se carece de la tradición que existe para otras plataformas, especialmente Microsoft Windows. Hasta ese momento, Sun Microsystems había utilizado un 
sistema propietario para sus aplicaciones Java. Sin embargo, ya han comenzado a aparecer otras alternativas (2)

- jHelp, de Moondog Software.

- Jelp, de CreativeSoft.

- Helplet, de VisualTek.

- Oracle Help for Java —en adelante OHJ— de Oracle Corporation y ...

- La especificación JavaHelp de Sun Microsystems.

\subsection{Sun JavaHelp}

La versión provisional de esta especificación se anunció el 10 de febrero de 1998. JavaHelp va a ser parte del JDK 1.2, aunque inicialmente el ritmo de actualizaciones será más rápido para JavaHelp que para el kit de desarrollo. La especificación se compromete a mantener el carácter multiplataforma propio del lenguaje Java y la independencia respecto a navegadores específicos.

De la misma forma que ocurrió con el sistema WinHelp de Microsoft, los fabricantes de herramientas para la creación de documentación técnica (ForeFront, CreativeSoft, Blue Sky Software, Quadralay, etc) han hecho público su compromiso de soportar la nueva especificación y ofrecer entornos que faciliten la creación y administración de hiperdocumentos.

\section{Evaluación}

En los siguientes apartados se describen las conclusiones de la evaluación de las alternativas enumeradas en el apartado anterior. En la evalución se ha atendido a los criterios que recoge la figura 1 .

En los siguientes apartados se describe el comportamiento de las aplicaciones evaluadas en cada uno de los puntos anteriores.

\subsection{Basados en estándares abiertos (HTML, XML, etc.)}

- Oracle Help for Java toma como base el lenguaje HTML versión 3.2 y la especificación Microsoft SiteMap para generar las tablas de contenidos y los índices de palabras clave. Esto último le separa de la situación de estándar, pero garantiza la compatibilidad entre los sistemas de ayuda creados para aplicaciones Java y los creados para aplicaciones Windows.

- Jelp se basa en archivos rtf, hpj y bmp. Es decir, los mismos archivos fuente que se pueden utilizar para generar un sistema de ayuda WinHelp pueden ser reutilizados en un sistema de documentación para Java, si bien no todas las características disponibles con WinHelp están disponibles en este sistema. 


\section{Criterios evaluación}

Basados en estándares abiertos (html, xml, etc.)

Facilidad del proceso de producción (disponibilidad de herramientas y asistentes)

Mecanismos de acceso a la información (tablas de contenidos, búsqueda en texto completo, índices de palabras claves, etc.)

Posibilidades de edición básicas (anotaciones públicas y privadas, marcas de lectura - bookmarks -, hiperenlaces, edición del texto, etc.)

Facilidad para implementar la sensibilidad al contexto

Soporte a formatos gráficos y multimedia

Capacidad de formatear el documento (tablas, marcos o frames, posicionamiento, zonas fijas y desplazables, estilos de letra, etc.)

Estructura lógica del hiperdocumento

Tratamiento de Information Types

Mecanismos de compresión

Modularidad de componentes (crear un único hiperdocumento a partir de dos o más hiperdocumentos, elegir el visor de documentos, indexador full-text, etc.)

Documentación y soporte.

Fig. 1. Criterios de evaluación

- JHelp y Helplet toman como base la versión 2.0 de HTML, al que añaden marcas propietarias para poder generar los índices de palabras clave. Aceptan los formatos de imagen gif y jpg.

- Finalmente, JavaHelp se basa en la misma versión de HTML que OHJ, la 3.2 y trabaja con archivos gráficos GIF y JPG. La utilización de archivos XML para la tabla de contenidos y los índices de palabras clave le sitúan en una posición muy similar a OHJ. De hecho, estos dos sistemas obtendrían al mejor evaluación en cuanto al respeto de dichos estándares. 


\subsection{Facilidad proceso producción}

En este apartado se evalúan en qué medida los sistemas soportan el proceso de creación y edición del contenido del hiperdocumento (entornos gráficos, gestión de librerías de imágenes, posibilidad de trabajar con el portapapeles, plantillas predefinidas, compilación, etc.). Es decir, se evalúa si incluye un entorno de authoring:

- OHJ facilita el proceso de creación de índices en texto completo y de palabras clave así como de tablas de contenidos mediante unos asistentes (wizards). De todas formas, los archivos deben haberse creado inicialmente con otra herramienta de authoring (3).

- Jelp incorpora un asistentes para crear el hiperdocumento en tres fases. Como en el caso anterior, los archivos fuentes deben haber sido creados con otra aplicación y Jelp no incorpora ninguna herramienta que facilite su creación.

- jHelp dispone únicamente de un asistente para crear índices de palabras clave. Éstas deben marcarse previamente en el texto de los archivos HTML. Como en los casos anteriores, jHelp carece de un entorno de authoring propiamente dicho.

- Finalmente, JavaHelp tampoco ofrece herramientas de authoring, aunque existe el compromiso por parte de los principales fabricantes de herramientas para la creación de documentación en línea de soportar esta especificación de la misma forma que han venido haciendolo con Microsoft WinHelp.

\subsection{Mecanismos de acceso a la información}

La facilidad para acceder a grandes volúmenes de información distribuídos en una estructura hipertextual es un factor clave en el éxito de un sistema de documentación en línea.

Tradicionalmente, los mecanismos de acceso utilizados en los entornos de edición electrónica son las tablas de contenidos desplegables, los índices de palabras clave asignadas a los distintos temas o páginas del hiperdocumento y la búsqueda en texto completo.

- De todas las alternativas evaluadas, únicamente OHJ y JavaHelp ofrecen estos tres mecanismos de acceso. Las tablas de contenidos y los índices de palabras clave de $\mathrm{OHJ}$ se basan en la misma estructura y tipo de archivos que los utilizados por el sistema HTML-Help de Microsoft. En el caso de JavaHelp se utilizan archivos XML.

- Jelp carece de búsqueda en texto completo. Para generar la tabla de contenidos y el índice, se utiliza el mismo procedimiento que en WinHelp: un

Scire. $4: 1$ (en.-jun. 1998) 131-145. 
archivo en texto plano con extensión .cnt que contiene la estructura de la tabla de contenidos y palabras clave codificadas en forma de notas a pie de página.

- Finalmente, jHelp es el sistema que recibe peor puntación en este punto. El único medio para buscar información es un índice de palabras clave.

\subsection{Posibilidades de edición básicas}

Por edición básica nos referimos a la posibilidad de añadir anotaciones al texto de un hiperdocumento, definir marcas de lectura — entradas de menú desde las que podemos acceder a un tema específico sin necesidad de recorrer la estructura del hiperdocumento- - y añadir nuevos hiperenlaces.

Otras posibilidades de edición más sofisticadas, como añadir o modificar texto al hiperdocumento, no se consideran.

Ninguno de los sistemas evaluados ofrece estas posibilidades básicas, aunque en todos los casos están anunciadas para versiones posteriores.

Por otra parte, en el caso de los sistemas que se basan en un mecanismo de compilación, la posibilidad de editar el hiperdocumento se hace imposible de no ser que el fabricante ofrezca gratuitamente herramientas para decompilar. Desde esta perspectiva, OHJ — que se basa en texto HTML no compilado—, sería el único que ofrecería posibilidades de edición y personalización reales.

\subsection{Sensibilidad al contexto}

Con este término hacemos referencia a la posibilidad de que el sistema presente una página o fragmento específico del hiperdocumento en pantalla, en respuesta a una acción ejecutada por el usuario desde la aplicación software (por ejemplo, seleccionar una opción de menú, presionar una tecla, etc.).

Todos los sistemas de documentación en línea ofrecen esta posibilidad. Por lo tanto, se evaluará en qué medida en fácil implantar la sensibilidad al contexto y qué ejemplos documentados facilitan los distintos fabricantes:

- OHJ y JavaHelp ofrecen la documentación más exhaustiva, con numerosos ejemplos.

- Jelp ofrece una buena documentación.

- La de VisualTek y jHelp no es tan completa como la del resto de productos evaluados.

\subsection{Formatos gráficos y multimedia}

En este punto se evalúa los formatos gráficos y multimedia soportados por estos sistemas: 
- OHJ, JavaHelp, Helplet y jHelp aceptan los formatos utilizados en la edición electrónica para internet: GIF y JPG.

- Jelp trabaja con formatos gráficos propios de WinHelp: BMP y WMF.

Respecto a los archivos multimedia, ningún sistema permite un tratamiento adecuado de la información audio o video. Jelp únicamente acepta archivos de sonido AU (no soporta video), y OHJ presenta las limitaciones propias del navegador HotJava, el cual utiliza para mostrar el contenido del hiperdocumento.

\subsection{Capacidades de formateo}

En este punto se evalúa la posibilidad de formatear el hiperdocumento utilizando distintos tipos de letra, ajustes, tablas, marcos, etc.:

- JavaHelp y OHJ soportan la versión 3.2 del lenguaje HTML (con tablas y marcos).

- JHelp y Helplet presentan serias limitaciones: las propias de la versión 2.0 del lenguaje HTML.

- Jelp, basado en el formato RTF, permite trabajar con las posibilidades de formateo disponibles en WinHelp, incluyendo zonas fijas y desplazables y ventanas emergentes. De todas formas, en la fase de evaluación se han observado ciertas limitaciones en el formateo de párrafos.

\subsection{Estructura del hiperdocumento}

En todos los casos los documentos están compuestos por una serie de temas o páginas enlazados. Véamoslo con más detalle:

- JHelp, Jelp y Helplet comprimen todos los archivos fuente utilizados en el hiperdocumento en una única clase. En el caso de Jelp no se reconocen los enlaces dirigidos a zonas intermedias de una página.

- La versión evaluada de OHJ no permite compilar los archivos fuente en un único archivo. Si bien esto facilita las posibilidades de edición (el texto podrá modificarse con cualquier editor HTML o ASCII), dificulta la gestión de los documentos que estan constituidos por un gran número de archivos.

- JavaHelp permite optar o no por la compresión de los archivos fuente en un único fichero.

\subsection{Information types}

Con este término designamos la capacidad de mostrar u ocultar parte de los temas de un hiperdocumento - así como sus correspondientes entradas en el índice y en la tabla de contenidos-, dependiendo de unas variables facilitadas por el usuario en el momento de acceder al hiperdocumento. 
Ninguno de los sistemas evaluados soporta los information types ni lo tienen previsto para próximas versiones.

Señalaremos que WinHelp nunca llegó a ofrecer esta posibilidad, y que hemos tenido que esperar al sistema HTML-Help de Microsoft para poder trabajar con ellos.

\subsection{Mecanismos de compresión}

La versión actual de $\mathrm{OHJ}$ no compila, si bien para un futuro está anunciada esta funcionalidad. Javahelp permitirá compilar o no los archivos en un único archivo, con un comportamiento igual al de OHJ.

El resto de sistemas (Jelp, jHelp y Helplet) reúnen todos los archivos fuente en una única clase Java comprimida.

\subsection{Modularidad de componentes}

- En el momento actual, ningún sistema permite trabajar con documentos compuestos, si bien Jelp se acerca a ello parcialmente, pues al menos permite enlazar dos archivos compilados diferentes.

- Los componentes de OHJ y JavaHelp (visor, indexador, etc.) podrían ser reemplazados por otros distintos.

- Jelp, Helplet y jHelp dependen de un sistema de compilación/visualización propietario y que, al menos a priori, no puede ser reemplazado.

\subsection{Documentación y soporte}

OHJ y JavaHelp ofrecen la documentación más completa. La documentación de Helplet no alcanza el nivel de detalle de las anteriores. Jelp y jHelp se sitúan a medio camino entre las anteriores.

\section{Conclusiones}

Para concluir, podemos señalar que se trata de sistemas todavía inmaduros:

- JavaHelp está destinado a convertirse en un estándar de facto.

- Jelp cambiará la orientación de su producto para ofrecer un entorno de producción de sistemas de documentación basados en la especificación de JavaHelp. De esta forma, su papel será similar al que han desempeñado fabricantes como Blue Sky o ForeFront respecto a Microsoft WinHelp.

- OHJ se mantendrá como el sistema de documentación en línea para soluciones Intranet/Internet desarrolladas a partir de productos Oracle.

- En el caso de jHelp y Helplet su evolución resulta más confusa.

Finalmente, no debe dejar de considerarse la posibilidad de utilizar Microsoft HTML-Help con aplicaciones Java, ya que: 
- Ofrece cierta independencia respecto al navegador con el que se accede a la documentación (únicamente se perdería la búsqueda en texto completo si se trabajase con un navegador que no fuese Internet Explorer).

- Comparte la base y estructura de OHJ.

- Esto le convertiría en una alternativa que merecería más atención si no resultase tan complejo hacer las llamadas contextuales a este sistema con aplicaciones no comercializadas por Microsoft, y esta empresa no hubiese detenido el desarrollo de HTML-Help basado en applets Java.

\section{Notas}

(1) Este último punto es importante si pensamos en la creciente importancia que han adquirido las aplicaciones software distribuidas. Estas aplicaciones son el resultado de ensamblar objetos que se ejecutan en ordenadores diferentes de forma transparente para el usuario. Los sistemas de documentación en línea diseñados para este tipo de aplicaciones deben cumplir los mismos requisitos de transparencia de acceso.

(2) A estas alternativa debe unirse, con cierta restricciones, la posibilidad de utilizar el sistema HTML-Help de Microsoft, con las restricciones que impone la utilización de los applets Java en lugar de los controles ActiveX originales.

(3) La versión evaluada no permitía compilar los distintos archivos en un único archivo. 
144 Ricardo Eito Brun

\section{Apéndices}

\subsection{Tabla de características}

\begin{tabular}{|c|c|c|c|c|c|c|c|}
\hline & WinHelp & ОНJ & Jelp & jHelp & Helplet & $\begin{array}{l}\text { Java } \\
\text { Help }\end{array}$ & $\begin{array}{c}\text { HTML } \\
\text {-Help }\end{array}$ \\
\hline Html & & $\mathrm{X}$ & & $\mathrm{X}$ & $\mathrm{X}$ & $\mathrm{X}$ & $\mathrm{x}$ \\
\hline .Rtf & $X$ & & $X$ & & & & \\
\hline Incluye Authoring & & & & & & & $\mathrm{X}$ \\
\hline Asistentes & & $\mathrm{X}$ & $\mathrm{X}$ & $\mathrm{X}$ & & & $\mathrm{X}$ \\
\hline Tablas contenidos & $\mathrm{X}$ & $\mathrm{X}$ & $\mathrm{X}$ & & $\mathrm{X}$ & $\mathrm{X}$ & $\mathrm{X}$ \\
\hline $\begin{array}{l}\text { Indice palabras } \\
\text { clave }\end{array}$ & $\mathrm{X}$ & $\mathrm{X}$ & $\mathrm{X}$ & $\mathrm{X}$ & & $\mathrm{X}$ & $\mathrm{X}$ \\
\hline Búsqueda full-text & $\mathrm{X}$ & $\mathrm{X}$ & & & & $\mathrm{X}$ & $\mathrm{X}$ \\
\hline $\begin{array}{c}\text { Anotaciones } \\
\text { públicas }\end{array}$ & $\mathrm{X}$ & & & & & & \\
\hline $\begin{array}{c}\text { Anotaciones } \\
\text { privadas }\end{array}$ & & & & & & & \\
\hline Bookmarks & $X$ & & & & & & \\
\hline \multicolumn{8}{|l|}{ Crear hiperenlaces } \\
\hline Multimedia & $\mathrm{X}$ & & $\mathrm{X}$ & & & & $\mathrm{X}$ \\
\hline Tablas & & $X$ & & & & $\mathrm{X}$ & $\mathrm{X}$ \\
\hline Marcos (frames) & & $\mathrm{X}$ & & & & & $\mathrm{X}$ \\
\hline \multicolumn{8}{|l|}{$\begin{array}{c}\text { Posicionamiento } \\
\text { texto }\end{array}$} \\
\hline Zonas fijas & $\mathrm{X}$ & & $\mathrm{X}$ & & & & \\
\hline Zonas desplazables & $\mathrm{X}$ & $\mathrm{X}$ & $\mathrm{X}$ & $\mathrm{X}$ & $\mathrm{X}$ & $\mathrm{X}$ & $\mathrm{X}$ \\
\hline Formato texto & $\mathrm{X}$ & $\mathrm{X}$ & $\mathrm{X}$ & & & $\mathrm{X}$ & $\mathrm{X}$ \\
\hline Formato párrafo & $\mathrm{X}$ & & $\mathrm{X}$ & & & & \\
\hline Information types & & & & & & & $\mathrm{X}$ \\
\hline Compresión & $\mathrm{x}$ & $\mathrm{X}$ & $\mathrm{X}$ & $\mathrm{x}$ & $\mathrm{x}$ & $\mathrm{x}$ & $\mathrm{x}$ \\
\hline Modularidad & $\mathrm{X}$ & $\mathrm{X}$ & $X$ & & & $\mathrm{X}$ & $\mathrm{X}$ \\
\hline
\end{tabular}

Scire. $4: 1$ (en.-jun. 1998) 131-145. 


\subsection{Evolución del lenguaje Java}

\begin{tabular}{|c|c|}
\hline 23 Mayo 1995 - Sun lanza Java & \multirow[t]{7}{*}{1995} \\
\hline Agosto - Netscape decide trabajar con Java & \\
\hline Octubre - Oracle, Macromedia, Borland y Toshiba obtienen licencias de Java & \\
\hline Noviembre - Spyglass obtiene licencias & \\
\hline Diciembre & \\
\hline Adobe, Symantec, Intel, AT\&T y Silicon Graphics obtienen licencias & \\
\hline Sun y Netscape anuncian JavaScript. & \\
\hline Enero - Sun crea JavaSoft como una nueva unidad de negocio & \multirow[t]{16}{*}{1996} \\
\hline 23 Enero 1996 - Sun libera Java Development Kit JDK 1.0 & \\
\hline Febrero - Sun anuncia el acceso a bases de datos JDBC & \\
\hline Marzo - Microsoft obtiene licencias de Java. & \\
\hline Abril & \\
\hline NEC, NCSA, SCO, Apple y Tandem obtienen licencias. & \\
\hline Apple, IBM, Microsoft, Novell y SCO, incluyen Java en sus sistemas operativos. & \\
\hline Mayo - Fujitsu, Hewlett-Packard y Sybase obtienen licencias. & \\
\hline Julio - GemStone obtiene licencias Java & \\
\hline Septiembre - DEC y Eastman Kodak adquieren licencias & \\
\hline Octubre - Marimba lanza CastaNet & \\
\hline Diciembre & \\
\hline Sun anuncia JDK 1.1. & \\
\hline Symantec Corp. lanza la herramienta de desarrollo Visual Cafe & \\
\hline En Internet World se anuncia la 100\% Pure Java Initiative. & \\
\hline Versión beta de JavaBeans Development Kit. & \\
\hline Enero & \multirow[t]{6}{*}{1997} \\
\hline Sun libera JavaBeans Development Kit & \\
\hline VisualTek Solutions lanza Rendezvous, aplicación groupware desarrollada completamente en Java. & \\
\hline Julio - Sun lanza Java Web Server 1.0.1. & \\
\hline Agosto - Sun y Netscape liberan la versión 2 de las Java Foundation Classes. & \\
\hline Desarrollo continuado y atención creciente a Java... & \\
\hline 5 Mayo 1998 - Sun hace pública la primera versión de JavaHelp & 1998 \\
\hline
\end{tabular}

Scire. $4: 1$ (en.-jun. 1998) 131-145. 